\title{
Involvement of Kytococcus schroeteri in a case of prosthetic valve endocarditis
}

\author{
Antonella D’Andria', Lucio Avallone', Luigi Cinquanta', Luigi Greco², Simona Marino', \\ Angelo Massari' \\ 'Servizio di Medicina di Laboratorio \\ ${ }^{2}$ Malattie Infettive;A.O. “S. Giovanni di Dio e Ruggi d’Aragona"- Salerno
}

Key words: Endocarditis, micrococci, blood culture

Implicazione di Kytococcus schroeteri in un caso di endocardite da valvola protesica

\section{SUMMARY}

We report a fourth case of prosthetic valve endocarditis caused by the newly described micrococcal species: Kytococcus schroeteri.

A 38-year old male was admitted to our hospital with suspected prosthetic valve endocarditis. In three blood cultures and in the prosthetic heart valve culture grew the same type of microrganism, initially identified as Micrococcus luteus.

Presuntive assignment to the genus Kytococcus was suggested by the arginina dihydrolase activity and by the resistence to penicillin, oxacillin and methicillin, characteristics which are not shared by other micrococci.

Further biochemical tests confirmed that the isolates belonged to the genus Kytococcus (negative reaction for oxydase, esculin and urease, positive for catalase and arginine dihydrolase) species schroeteri (alpha-glucosidase positive).

The patient was treated with vancomycin, gentamicin and rifampicin. After the valve replacement, on the $20^{\text {th }}$ day of hospitalization, the patient's biological parameters returned to normal values, and after one mounth the resolution of the disease was completed.

The diagnosis of four cases by K. schroeteri endocarditis, described within a short period of time (2003-2006), might indicate a specific pathogenicity of this new species.

In case of endocarditis diagnosis, the suspect of the genus Kytococcus involvement should be considered each time the aerobic Gram positive cocci, with arginina dihidrolase activiy and the resistence to penicillin, oxacillin and methicillin, are isolated by significative biological samples.

Received February 19, 2007

Accepted November 19, 2007

\section{INTRODUZIONE}

L'endocardite batterica è una infezione che colpisce la superficie interna del muscolo cardiaco o le valvole cardiache native o artificiali; viene classificata come "acuta" o "subacuta-cronica" sulla base del tempo di incubazione, della severità della presentazione e della progressione clinica. In modo caratteristico l'endocardite batterica si manifesta con una vegetazione localizzata nel sito dell'infezione che risulta composta da piastrine, fibrina, microrganismi e cellule infiammatorie. Raramente l'endocardite batterica colpisce soggetti sani, molto più frequentemente insorge in soggetti affetti da cardiopatie.

Le valvole protesiche rappresentano la sede nel 7$25 \%$ (5) dei casi di endocardite infettiva: sono infatti ad elevato rischio di infezione, in particolare nei tre mesi successivi all'impianto chirurgico.
I casi di endocardite che si manifestano entro due mesi dall'intervento vengono definiti "precoci" e sono più frequentemente causati da microrganismi acquisiti in ospedale durante l'intervento o immediatamente dopo. I casi che si verificano dodici mesi dopo l'intervento vengono definiti "tardivi" e sono dovuti a microrganismi acquisiti in comunità e diffusi per via ematogena. Invece, quelli che si verificano tra i due e i dodici mesi dall'intervento possono rappresentare l'espressione o di una contaminazione perioperatoria da parte di microrganismi a bassa virulenza, o di una contaminazione da microrganismi acquisiti dalla comunità e diffusi per via ematogena.

Negli ultimi anni gli stafilococchi, e in particolare lo Staphyloccoccus aureus, hanno superato gli streptococchi viridanti come più comune causa di endocardite batterica. In più gli stafilococchi coa-

\section{Corresponding author: Antonella D'Andria}

Servizio di Medicina di Laboratorio - A.O. "S. Giovanni di Dio e Ruggi d'Aragona" - Via San Leonardo - 84I00 Salerno Tel.: 089672595 - E-mail: antoniad@alice.it 
gulasi-negativi sono quelli più frequentemente isolati in casi di endocarditi su valvole protesiche a esordio precoce, mentre sono solo occasionalmente isolati in caso di endocarditi in valvole native. Gli streptococchi che più frequentemente sono isolati da pazienti con endocardite sono Streptococcus sanguis, $S$. bovis, $S$. mutans e $S$. mitis. Enterococcus spp. è frequentemente associato ad endocarditi nosocomiali e solitamente risulta più resistente al trattamento medico. Eventi rari sono le endocarditi polimicrobiche che vengono frequentemente riscontrate in soggetti dediti all'uso di droghe per via endovenosa (5). Rarissimi sono i casi di endocardite da Kytococcus, un micrococco di nuova descrizione: dal 2003 ad oggi sono stati descritti in letteratura solo tre casi di endocardite con sede su valvola protesica e con coinvolgimento di Kytococcus schroeteri.

Noi riportiamo il quarto caso di endocardite su valvola protesica causata da questo ancora poco conosciuto batterio.

Il genere Kytococcus è classificato (2) nella famiglia Dermatophilaceae subordine Micrococcineae e comprende, al momento, due sole specie (8): $K$. sedentarius, un normale saprofita della cute e delle mucose umane, e K. schroeteri, il cui habitat rimane ancora sconosciuto.

Il genere Kytococcus comprende cocchi Gram positivi, non-capsulati, non mobili, aerobi e catalasi positivi che differiscono dagli altri micrococchi perché mostrano attività di arginina deidrossilasi e resistenza verso penicillina, oxacillina e meticillina (2). I casi di endocardite da $K$. schroeteri riportati in letteratura impongono una maggiore attenzione nei confronti di questo germe come possibile agente eziologico di endocarditi.

\section{CASO CLINICO}

Paziente maschio di 39 anni giunge alla nostra osservazione nell'Aprile 2005 per persistenti episodi febbrili, ricorrenti da circa quattro mesi. Il paziente, affetto da valvulopatia aortica, nel Dicembre 2004 è stato sottoposto presso altro nosocomio ad intervento chirurgico per sostituzione della valvola aortica e dell'aorta ascendente (intervento di Bentall).

Al ricovero il paziente presenta febbre (temperatura $39^{\circ} \mathrm{C}$ ), affaticamento e dispnea. All'esame obiettivo non si evidenziano rash, petecchie o linfoadenopatia. Si evidenzia respiro affannoso, soffio olosistolico, tracce di edema su entrambe le estremità, frequenza cardiaca elevata (100 battiti al minuto) e pressione arteriosa di 100/70 $\mathrm{mm} \mathrm{Hg}$. I parametri di laboratorio al ricovero sono i seguenti: emoglobina $(\mathrm{Hb}) 10.9 \mathrm{~g} / \mathrm{dL}$, globuli bianchi (GB) $15.42 \times 10^{3} \mathrm{u} / \mathrm{L}$, neutrofili $12.16 \times$
$10^{3} \mathrm{u} / \mathrm{L}$, velocità di eritrosedimentazione (VES) $83 \mathrm{~mm} / \mathrm{h}$, proteina C-reattiva (PCR) $8.3 \mathrm{mg} / \mathrm{dL}$. I test di funzionalità epatica, renale risultano nella norma come gli elettroliti plasmatici.

Nelle 24 ore successive al ricovero viene praticato un set completo di emocolture e una ecocardiografia transesofagea.

L'ecocardiografia transesofagea evidenzia una dissezione della protesi valvolare aortica su cui viene evidenziata una vegetazione suggestiva per endocardite.

Dopo consulenza infettivologica, viene praticato empiricamente un trattamento endovenoso con rifampicina (60mg/die), gentamicina ( $160 \mathrm{mg} / \mathrm{die})$ e vancomicina ( $2 \mathrm{gr} / \mathrm{die}) \mathrm{e}$, a due giorni dal ricovero (la temperatura corporea era di $37^{\circ} \mathrm{C}$ ), il paziente viene sottoposto ad intervento chirurgico per la sostituzione della protesi valvolata infetta e impianto di condotto pericardico porcino valvolato con protesi biologica.

Il materiale protesico infetto, rimosso durante l'intervento chirurgico, viene inviato al laboratorio di Microbiologia per la coltura.

Il decorso post-operatorio risulta privo di problemi. La terapia antibiotica endovenosa con rifampicina, vancomincina e gentamicina viene protratta per tre settimane e, dopo quattro settimane, tornati normali i parametri di laboratorio (GB $8.20 \mathrm{x}$ $10^{3} / \mathrm{uL}$, neutrofili $5.42 \times 10^{3} / \mathrm{uL}$, VES $33 \mathrm{~mm} / \mathrm{h}$, PCR $0.3 \mathrm{mg} / \mathrm{dL}$, emocolture negative), il paziente viene dimesso.

Microbiologia - Il set di emocolture (tre prelievi praticati a intervalli di 30 minuti) e il materiale protesico con la vegetazione, sono stati processati con sistema Bactec 9240 della Becton Dickinson utilizzando i flaconi Plus Aerobic/F e Plus Anaerobic/F.

Dopo 48 ore di incubazione a $35^{\circ} \mathrm{C}$, dai tre flaconi Plus Aerobic/F utilizzati per l'emocoltura in cui viene rilevata crescita batterica vengono preparati i vetrini per l'esame microscopico diretto e allestite le subcolture sui seguenti terreni: 5\% Sheep Blood Agar, Mannitol Salt Agar e Mac Conkey Agar. L'esame microscopico diretto rivela la presenza di cocchi non mobili, la subcoltura su 5\% Sheep Blood Agar dopo 48 ore di incubazione a $37^{\circ} \mathrm{C}$, rivela colonie circolari a lenta crescita (diametro di circa 2-4 mm), giallastre, mucose, convesse e prive di emolisi (figura I).

La colorazione di Gram, eseguita su vetrino allestito dalle colonie, rivela cocchi Gram positivi disposti prevalentemente a paia e a tetradi, raramente i cocchi si presentano in cluster (figura II). L'attività della catalasi è stata testata su vetrino osservando la formazione di bollicine dopo aver mescolato una sospensione della coltura con una 
goccia di perossido di idrogeno al 3\%. L'attività dell'ossidasi è stata testata con il reagente Oxidase Reagent Droppers (BD) basato sulla produzione dell'enzima indofenolo ossidasi. Le altre caratteristiche biochimiche (alofilia, fosfatasi alcalina, arginina deidrossilasi, $\alpha$-glicosidasi, ureasi, indolo, lisina e ornitina decarbossilasi, esculina, riduzione dei nitrati, utilizzo dei carboidrati, comportamento nei confronti di bacitracina, lisozima e lisostafina) descritte di seguito sono state testate utilizzando i substrati presenti nelle card GPI, GNI e ANI del sistema Vitek, e nelle gallerie dei sistemi manuali API 20A e API ID 32 STAPH (bioMérieux).

Il microrganismo isolato è strettamente aerobio (nessuna crescita nelle sub-colture su 5\% Sheep Blood Agar realizzate in condizioni di microaerofilia e anaerobiosi), cresce in presenza di $\mathrm{NaCl}$ al $7 \%$, presenta attività di catalasi, fosfatasi alcalina e arginina deidrossilasi. Non presenta attività di $\alpha$-glicosidasi, ossidasi e ureasi. La reazione all'indolo è negativa, non vi è attività di lisina $\mathrm{e}$ ornitina decarbossilasi, non vi è idrolisi dell'esculina, né riduzione dei nitrati. L'isolato è inoltre metabolicamente inerte per quanto riguarda la produzione di acidi a partire dai carboidrati ed è sensibile alla bacitracina, al lisozima ma resistente alla lisi da lisostafina.

Il test di sensibilità agli antibiotici, allestito su Muller-Hinton Agar viene eseguito utilizzando il metodo di diffusione in agar da disco, (KirbyBauer), e interpretato secondo i criteri CLSI ( un tempo NCCLS) 2006 per gli stafilococchi (4).

L'isolato è resistente a: ampicillina, meticillina, penicillina, eritromicina, meropenem, oxacillina, e sensibile a: linezolid $(40 \mathrm{~mm})$, amoxicillina/acido clavulanico $(20 \mathrm{~mm})$, rifampicina $(40 \mathrm{~mm})$, cloramfenicolo $(30 \mathrm{~mm})$, teicoplanina $(21 \mathrm{~mm})$ e vancomicina $(24 \mathrm{~mm})$.

Dopo 48 ore di incubazione, anche la coltura in flacone Plus Aerobic/F della protesi valvolare aortica con la vegetazione risulta positiva. La subcoltura su 5\% Sheep Blood Agar evidenzia lo sviluppo di colonie identiche a quelle già descritte $\mathrm{e}$ dal Gram eseguito dalla subcultura si conferma lo sviluppo di cocchi Gram positivi. Tutti gli isolati mostrano identico biotipo e stesso pattern di suscettibilità e vengono quindi, considerati appartenenti allo stesso ceppo.

\section{COMMENTO}

In una fase iniziale del nostro lavoro di indagine microbiologica, l'isolato era stato identificato come appartenente al genere Micrococcus sulla base delle caratteristiche morfologiche delle colonie e sulla sua incapacità di crescita in condizioni di anaerobiosi. Questa ipotesi era stata suffragata dall'identificazione ottenuta dopo 48 ore di incubazione, dalla galleria del sistema API ID 32 STAPH della bioMérieux che aveva identificato il microrganismo come Micrococcus luteus. Il sospetto che il nostro isolato appartenesse al genere Kytococcus è venuto dal riscontro che il ceppo mostrava resistenza a penicillina, oxacillina e meticillina, caratteristiche non mostrate da Micrococcus luteus né da altri micrococchi. Un riesame attento dei test biochimici eseguiti fa considerare altamente probabile che l'isolato appartenga al genere Kytococcus (reazione negativa per ossidasi, esculina, ureasi e positiva per catalasi, fosfatasi alcalina e arginina deidrossilasi) specie schroeteri (reazione negativa alla alfa glicosidasi) (2). A tal proposito c'è da riferire che attualmente non sono disponibili metodi commerciali per l'identificazione di Kytococcus schroeteri, l'identificazione definitiva potrebbe scaturire solo dall'utilizzo di due metodologie: l'analisi del contenuto degli acidi grassi intracellulari o il sequenziamento completo del DNA ribosomiale 16S, entrambe non disponibili per uso diagnostico. Entrambe queste metodologie non sono attualmente in uso all'interno della nostra struttura e la scarsa vitalità mostrata dal ceppo isolato, non ha permesso l'invio presso un centro di riferimento, che procedesse alla identificazione molecolare.

La biologia del Kytococcus schroeteri non è del tutto definita, infatti rimane ancora sconosciuto l'habitat di questo microrganismo, così come restano non note le modalità di contaminazione $\mathrm{e}$ i suoi fattori di virulenza.

Kytococcus vuol dire letteralmente "cocco della pelle" e infatti l'habitat naturale di K.sedentarius (9) è rappresentato dalla cute e dalle mucose umane, ma è risultato vano il tentativo descritto da Le Brun et al. (6) di isolare K. schroeteri da queste sedi.

Nel nostro caso, come nel caso presentato da Becker et al. (2003) (3) si può ipotizzare una contaminazione perioperatoria, essendo i primi sintomi della patologia insorti nei tre mesi successivi all'intervento, a differenza dei casi presentati da Le Brun et al. (2005) (6) e da Basma et al. (2006) (1) nei quali s'ipotizza una diffusione del microrganismo per via ematogena da un sito incognito, in quanto i sintomi si sono manifestati rispettivamente dopo tre e dieci anni dall'intervento chirurgico. Riguardo al suo ruolo come patogeno, K. schroeteri, quando isolato da campioni clinici, probabilmente, non può essere più considerato semplicemente un contaminante o un opportunista, alla luce del caso clinico descritto e degli altri riportati in letteratura, ma deve essere considerato un patogeno vero e proprio, probabilmente caratterizzato da una bassa virulenza. 
Lo studio dei quattro casi di endocardite da $K$. schroeteri ci conduce anche ad un'altra importante considerazione, ovvero che è possibile che $K$. schroeteri presenti un particolare tropismo per $\mathrm{i}$ dispositivi cardiaci impiantati. Infatti, fino a questo momento, fatta eccezione per un caso di polmonite fatale in un paziente immunocompromesso (7), questo batterio è stato isolato solo da campioni biologici provenienti da pazienti affetti da endocardite e precedentemente sottoposti a interventi di cardiochirurgia protesica.

In conclusione, l'isolamento di cocchi Gram positivi, che mostrano attività di arginina deidrossilasi e resistenza a meticillina, oxacillina e penicillina, da campioni biologici significativi non va mai sottovalutata né ignorata, ma sempre ulteriormente indagata.

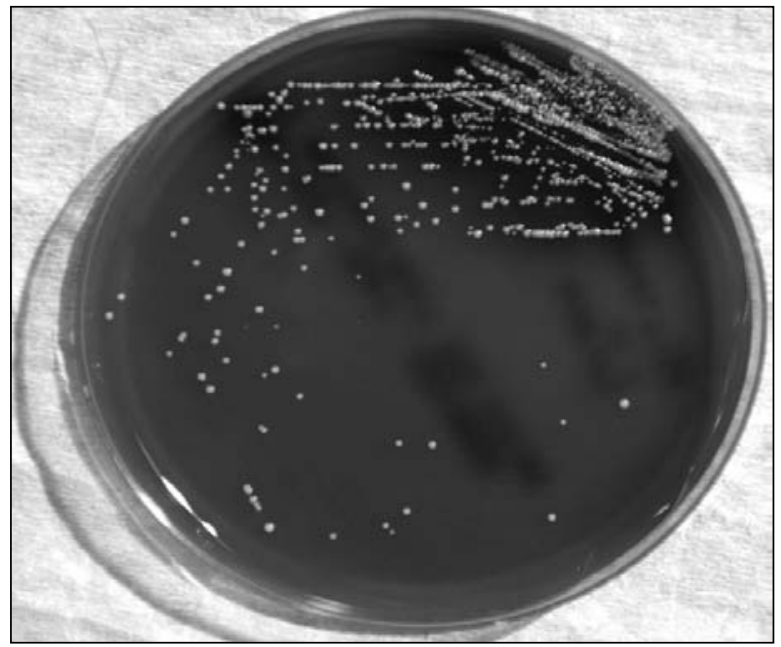

Figura I. Morfologia assunta dalle colonie di K. Schroeteri in $5 \%$ Sheep Blood Agar dopo 48 ore di incubazione a $37^{\circ} \mathrm{C}$ : si evidenziano colonie circolari a lenta crescita (diametro di circa 2-4 $\mathrm{mm}$ ), giallastre, mucose, convesse e prive di emolisi.

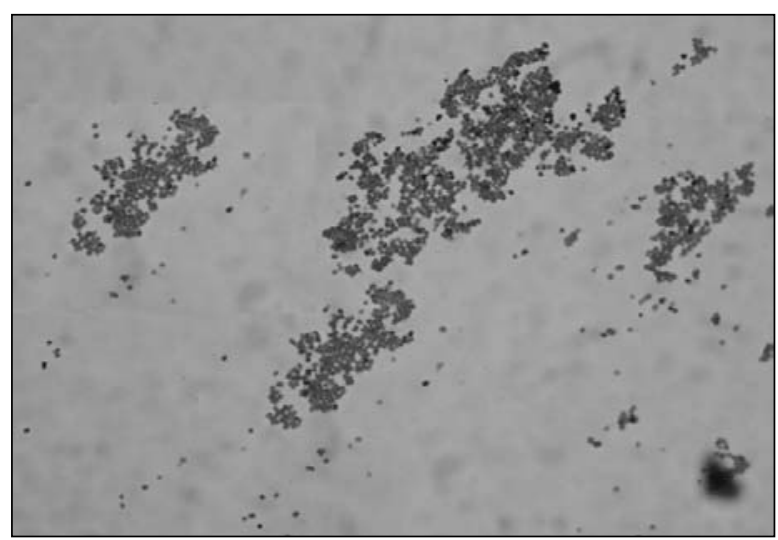

Figura II. K. schroeteri è un cocco Gram positivo prevalentemente disposto a paia e in tetradi

\section{BIBLIOGRAFIA}

1. Basma M, Boujelbène I, et al. Endocarditis due to Kytococcus schroeteri: case report and review of the literature. J of Clinical Microbiology 2006; 44 (3): 1187-9.

2. Becker K, Schumann P, Wullenweber J, et al. Kytococcus schroeteri spp nov., a novel Gram-positive actinobacterium isolated from a human clinical source. Int J Syst Evol Microbiol 2002; 52 (Pt 5): 1609-14.

3. Becker K, Wullenweber J, Odenthal HJ, et al. Prosthetic valve endocarditis due to Kytococcus schroeteri. Emerg Infect Dis 9 (2003); 1493-5.

4. Clinical and Laboratory Standards Institute. Performance Standards for Antimicrobial Disk Susceptibility Tests; Approved Standard - Ninth Edition; M2-A9 ed. Wayne (PA): The Committee; 2006;

5. Eleftherios Mylonakis, MD, Stephen B, Calderwood MD. Infective Endocarditis in Adults N Engl J Med 2001; (345); 1318-30.

6. Le Brune C, Bouet J, Gautier P, et al. Kytococcus schroeteri endocarditis. Emerging Infectious Diseases 2005; 11 (1).

7. Mohammedi I, Berchiche C, Becker K, et al. Fatal Kytococcus schroeteri bacteremic pneumonia. J Infect 2005 Aug; 51(2): E11-3.

8. Stackebrandt E, Koch C, Gvozdiak O, et al. Taxonomic dissection of the genus Micrococcus: Kocuria gen. nov., Nesterenkonia gen. nov., Kytococcus gen. nov., Dermacoccus gen. nov., and Micrococcus Cohn, 1872 gen. Emend. Int J Syst Bacteriol 45 (1995); 682-92.

9. Szczerba I, Krzeminski Z. Occurrence and number of bacteria from the Micrococcus, Kocuria, Nesterenkonia, Kytococcus and Dermacoccus genera on skin and mucous membranes in humans. Med Dosw Mikrobiol 2002; 55: 67-74. 